\title{
Antidepressants tested for everything from hot flashes to stroke
}

Antidepressants known as selective serotonin reuptake inhibitors (SSRIs) have become a fixture in many medicine cabinets ever since Prozac, the first such drug to receive US approval, hit the market in 1987. According to the health analytics firm SDI, in 2009 physicians wrote an estimated 52 million SSRI prescriptions for Americans aged 45 to 65 alone. Now, as evidenced by a flurry of papers this year, SSRIs are emerging as a possible treatment for a range of ailments from mood disorders to menopause.

"It's only a historical accident that these drugs were used for depression," says psychiatrist James Hudson, co-director of the Biological Psychiatry Laboratory at McLean Hospital in Belmont, Massachusetts.

Although the exact mechanism for SSRIs' action against depression remains unclear, the drugs are known to block the recycling of serotonin, making this important signaling chemical more available to trigger receptors at brain cell synapses.

Given that some people taking SSRIs for depression experience a loss of appetite, it's perhaps not surprising that one of the earliest alternative uses of these drugs was the treatment of bulimia. Indeed, when people with this eating disorder took Prozac (fluoxetine), they experienced a reduction in binge eating, leading to fewer bulimic episodes (Am. J. Psychiatry 155, 1756-1762, 1988).

Gerard Karsenty of Columbia University Medical Center in New York, says that SSRIs, which keep serotinin outside of cells, might actually decrease the amount of this neurotransmitter inside certain brain cells, and this intracellular drop in serotonin might suppress appetite. In one experiment his group conducted, mice genetically engineered to produce less serotonin in neurons ate about $25 \%$ less than their control counterparts (Cell 138, 976-989, 2009). A subsequent study published in January indicated that blocking serotonin signaling in certain neurons caused mice deficient in the appetite-suppressing hormone leptin to eat less (J. Exp. Med. 208, 41-52, 2011).

Also in January, a team of doctors led by Ellen Freeman of the University of Pennsylvania School of Medicine in Philadelphia reported that the SSRI Lexapro (escitalopram) may be an alternative to hormone replacement therapy to reduce hot flashes. In an eight-week trial involving 205 healthy, menopausal women, researchers found that $55 \%$ of women taking Lexapro experienced half as many hot flashes as before. By comparison, only $36 \%$ of those

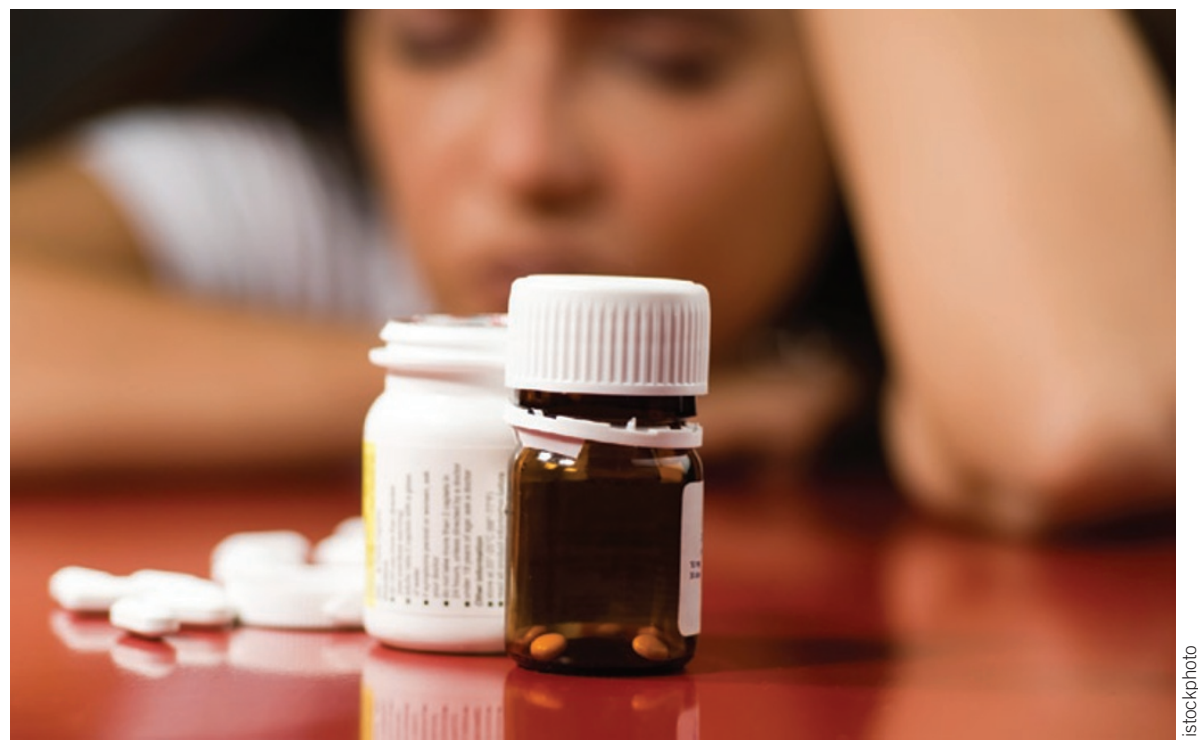

Down but not out: Researchers repurpose antidepressants to treat other diseases.

on placebo experienced the same reduction $(J$. Am. Med Assoc. 305, 267-274, 2011). SSRIs, however, may contribute to bone loss, so these drugs may not be a good alternative to hormone replacement therapy for menopausal women prone to osteoporosis (Arch. Intern. Med. 167, 1240-1245, 2007).

\section{Depressing on}

Given the important role of serotonin in the brain, studies have looked closely at the possibility of using SSRIs for neurological disorders and brain injury. In France, a team led by François Chollet from the University Hospital of Toulouse found that antidepressants might help stroke patients recover. In a trial involving 118 participants, the researchers found that subjects treated with Prozac five to ten days after stroke for three months showed a 50\% improvement in motor coordination compared to those who received physical therapy alone (Lancet Neurol. 10, 123-131, 2011). The study suggests that SSRI supplementation may benefit a larger number of patients than the standard clotbusting drugs, which must be administered within three hours of the stroke to be effective. "This trial opens up a new target-a neuronal target," Chollet says. "This may be a new way to treat patients."

Scientists from the University of Iowa in Iowa City, led by psychologist Leigh Beglinger, meanwhile, are conducting a multicenter phase 2 clinical trial of the SSRI Celexa (citalopram) for the treatment of Huntington's disease following independent results that showed that SSRIs slowed disease progression in mice (Ann. Neurol. 55, 590-594, 2004). Other groups hope to use SSRIs to reduce repetitive behaviors in a range of neurodevelopmental disorders including autism spectrum disorders and intellectual disability.

Large pharmaceutical companies, however, are not likely to get in the game. With many SSRIs soon to be off patent protection, "whether they have other indications or not, the generics will be used much more because managed care really does push the use of the drugs down," says analyst Ronny Gal of New York-based Sanford C. Bernstein.

Reformulation of these drugs is no guarantee that they will work for new indications, either. Last October, the drug company Neuropharm, based in Leatherhead, UK, closed when their low-dose formulation of Prozac failed to significantly reduce repetitive behaviors in children with autism in a phase 3 clinical trial.

Michelle Pflumm

\section{Correction}

In the February 2011 issue, the article entitled "Genzyme, though unique, could be a bellwether for US biotech" (Nat. Med. 17, 145, 2011) incorrectly stated that GlaxoSmithKline had acquired AstraZeneca. Although GlaxoSmithKline has acquired medicines from AstraZeneca in the past, the two companies never merged. The error has been corrected in the HTML and PDF versions of the article. 